\title{
The anti-cancer efficacy of curcumin scrutinized through core signaling pathways in glioblastoma
}

\author{
CHIN-CHENG SU ${ }^{1,2}$, MEI-JEN WANG ${ }^{3,4}$ and TSUNG-LANG CHIU ${ }^{2,5}$ \\ ${ }^{1}$ Division of General Surgery, Buddhist Tzu Chi General Hospital, No.707, Section 3, Chung-Yang Road; \\ ${ }^{2}$ Tzu Chi University, No.701, Section 3, Chung-yang Road; ${ }^{3}$ Department of Research, Buddhist Tzu Chi \\ General Hospital, No.707, Section 3, Chung-Yang Road; ${ }^{4}$ Tzu Chi College of Technology, No.880, Section 2, \\ Jian-Guo Road; ${ }^{5}$ Division of Neurooncology, Neuro-Medical Scientific Center, Buddhist Tzu-Chi \\ General Hospital, No.707, Section 3, Chung-Yang Road, Hualien, 97004, Taiwan, R.O.C.
}

Received March 16, 2010; Accepted April 29, 2010

DOI: 10.3892/ijmm_00000455

\begin{abstract}
Curcumin has been verified as an anti-cancer compound via multiple molecular targets. Its effective mechanisms include cell cycle arrest, inducing apoptosis, suppressing oncogenes, and enhancing tumor suppressor genes. The resistance of cells to chemotherapy, however, derives from the variable genetic aberration of cancer cells. Consequently, the core signaling pathways of glioblastoma have been explored to evaluate the efficacy of curcumin in proceeding through mutated genes in those pathways. In this study, the efficacy of curcumin was investigated in DBTRG cells. The cytotoxic ability was detected with MTT assay, and the influence of the cell cycle was checked with flow cytometry. The influence of the core signaling pathways was evaluated by Western blotting through the predominantly mutated proteins which included p53, p21, and cdc2 in the p53 pathway, CDKN2A/p16 and RB in the RB pathway, and EGFR, mTOR, Ras, PTEN, and Akt in the RTK-Ras-PI3K pathway. In addition, the apoptotic effect was determined by apoptosis-associated proteins $\mathrm{Bcl}-2, \mathrm{Bax}$, and caspase 3 . Curcumin exhibits superior cytotoxicity on glioblastoma in a dose- and time-dependent manner in the MTT assay. In the core signaling pathways of glioblastoma, curcumin either significantly influences the p53 pathway by enhancing p53 and p21 and suppressing cdc2 or significantly inhibits the RB pathway by enhancing CDKN2A/p16 and suppressing phosphorylated RB. In the apoptotic pathway, the Bax and caspase 3 are significantly suppressed by curcumin and the Giemsa stain elucidates apoptotic features of DBTRG cells as well. In conclusion, curcumin appears to be an effective anti-
\end{abstract}

Correspondence to: Dr Tsung-Lang Chiu, Division of Neurooncology, Neuro-Medical Scientific Center, Buddhist Tzu Chi General Hospital, No.707, sec. 3, Chung-Yang Road, Hualien City 97004, Taiwan, R.O.C.

E-mail: poluschiou@mail.com

Key words: curcumin, glioblastoma, core signaling pathway, apoptosis, genetic mutation glioblastoma drug through inhibition of the two core signaling pathways and promotion of the apoptotic pathway.

\section{Introduction}

Curcumin is a natural polyphenol compound and derived from several curcuma species, such as turmeric. It has been widely used in the human diet for centuries (1). However, its medical applications have been broadly investigated and explored, including its anti-cancer, anti-inflammation, and anti-angiogenesis activities. The anti-cancer effects of curcumin involve apoptosis of cancerous cells, cell cycle arrest, suppression of oncogenes, and promotion of tumor suppressor genes $(2,3)$. Furthermore, several anti-cancer mechanisms and pathways of curcumin on human cancers have been identified. As in the most prevalent cancers, curcumin can suppress EGFR of colon cancer cells in a dose-dependent manner and inhibit the activity of EPGR promoters through reducing DNA-binding activity of egr-1 (4). In prostate cancer, curcumin induces apoptosis through hypersensitivity to tumor necrosis factorrelated apoptosis-inducing ligand, which is mediated by inhibition of phospho-Akt and subsequently reductive transcription of nuclear factor (NF)- $\mathrm{B}$ (5). In breast cancer, curcumin inhibits cancer cell proliferation and migration by increasing the Bax to $\mathrm{Bcl}-2$ ratio and decreasing NF-kBp65 in a dose-dependent manner (6).

In malignant brain tumors, curcumin also exhibits its pleiotropic activity, which includes inducing cell cycle arrest at the G2/M stage through up-regulation of ING4, suppressing tumor growth by inhibition of AP-1 and NF- $\mathrm{BB}$, and inhibiting anti-apoptotic signaling by increasing the $\mathrm{Bax}$ to $\mathrm{Bcl}-2$ ratio (7-9). Nevertheless, most failure of cancer therapy in clinical practice is attributed to the polymorphism of cancer cells. To overcome this dilemma, a comprehensive exploration of genetic aberrations of cancer is not just helpful, but is required. The Cancer Genome Atlas have reported their research regarding the aberrations of DNA copy number, gene expression, and DNA methylation of glioblastoma multiforme (GBM) and concluded that there were three core aberrant signaling pathways, including RTK/RAS/PI3K, p53, and RB signaling pathways (10). Several genes were identified to be 
more frequently mutated in those pathways, such as EGFR and PTEN in the RTK pathway, CDKN2A(ARF) and TP53 in the p53 pathway, and CDKN2A(p16/INK4A) and CDKN2B in the RB pathway. In addition, adult malignant gliomas have been reviewed, and genetic alterations of different pathological types have been summarized. Among the anaplastic astrocytoma and GBM, the predominant mutated genes include EGFR, PTEN, MDM2, TP53, RB, and CDKN2A(INK4A), which are comparable to the discoveries of The Cancer Genome Atlas (11). To explore the ultimately effective antiglioblastoma activity of curcumin, we investigated the mechanism emphasized in the three mentioned core pathways.

\section{Materials and methods}

Preparation of reagents. Curcumin was purchased from Herbasin Co. (Shenyang, China), and aprotinin, antipain, sodium deoxycholate, leupeptin, propidium iodide (PI), sodium orthovanadate, Triton $\mathrm{X}-100$, Tris- $\mathrm{HCl}$, ribonuclease-A, and MTT [3-(4,5)-dimethylthiahiazo(-z-y1)-3,5-di-phenytetrazoliumromide] were obtained from Sigma Chemical Co. (St. Louis, MO, USA). Dimethyl sulfoxide (DMSO), potassium phosphates, and TE buffer were purchased from Merck Co. (Darmstadt, Germany). RPMI-1640 medium, fetal bovine serum (FBS), penicillin-streptomycin, trypsin-EDTA, and glutamine were obtained from Gibco BRL (Grand Island, NY, USA).

Preparation of human GBM cell line DBTRG. The human GBM cell line DBTRG was purchased from the Food Industry Research and Development Institute (Hsinchu, Taiwan). Cells were placed into $75-\mathrm{cm}^{3}$ tissue culture flasks and incubated with RPMI-1640 medium (Gibco BRL) containing $10 \%$ heat-inactivated fetal bovine serum, (FBS) (Gibco BRL), $1 \%$ penicillin-streptomycin $(10,000 \mathrm{U} / \mathrm{ml}$ penicillin; $10 \mathrm{mg} / \mathrm{ml}$ streptomycin), 1\% HEPES (Gibco BRL), and 1\% sodium pyruvate (Gibco BRL). Cells were grown at $37^{\circ} \mathrm{C}$ in humidified atmosphere $\left(5 \% \mathrm{CO}_{2}\right)$. All data presented in this report are from at least three independent experiments.

Effects of curcumin on the viability of DBTRG cells. The DBTRG cells were plated in a density of $1 \times 10^{4}$ cells/well and grown for $24 \mathrm{~h}$. Increased concentrations of curcumin 0,10 , 20 , to $30 \mu \mathrm{g} / \mathrm{ml}$ were added and cells were grown for 24 and $48 \mathrm{~h}$ at $37^{\circ} \mathrm{C}$, while the $0 \mu \mathrm{g} / \mathrm{ml}$-treated cells were set as control group and $0.2 \%$ DMSO (solvent) was added to these. For determining cell viability, after 24 and $48 \mathrm{~h}$ culture, the viability was evaluated by MTT assay and repeated four times. Briefly, DBTRG cells were seeded in a 96-well plate in a density of $1 \times 10^{4}$ cells/well and allowed to adhere overnight. After removing the medium, $200 \mu 1$ of fresh medium containing $10 \mathrm{mM}$ HEPES was added to each well, along with $20 \mu \mathrm{l}$ 3-(4,5-dimethylthiazol-2-yl)-2,5-diphenyl-2H-tetrazolium bromide (MTT) solution also added to each well. Then, the plate was incubated for $2 \mathrm{~h}$ at $37^{\circ} \mathrm{C}$ in the dark. After removing the medium, $100 \mu 1$ DMSO was added into each well. The absorbance was measured using an ELISA plate reader at $590 \mathrm{~nm}$.

Cell cycle analysis for DBTRG cells treated with curcumin by flow cytometry assay. The percentage of cells in sub-G1,
G0/G1, S, and G2/M phases were determined by flow cytometry as described in a previous report (12). Briefly, $2 \times 10^{6}$ DBTRG cells/10-cm dish were incubated with increased concentrations of curcumin, $0,8,16$, and $32 \mu \mathrm{g} / \mathrm{ml}$ for $24 \mathrm{~h}$ before cells were harvested by centrifugation. In addition, $2 \times 10^{6}$ DBTRG cells $/ 10-\mathrm{cm}$ dish were incubated with $16 \mu \mathrm{g} / \mathrm{ml}$ curcumin for $0,12,24$, and $48 \mathrm{~h}$. Cells, after being harvested, were washed with PBS then fixed gently (drop by drop) in $70 \%$ ethanol. Cells were put in an ice bath overnight then resuspended in PBS containing $40 \mu \mathrm{g} / \mathrm{ml}$ PI, $0.1 \mathrm{mg} / \mathrm{ml}$ RNase (Sigma), and $0.1 \%$ Triton $\mathrm{x}-100$. After $30 \mathrm{~min}$ incubation at $37^{\circ} \mathrm{C}$ in the dark, cells were analyzed with flow cytometry (Becton-Dickinson, San Jose, CA, USA) equipped with an argon laser at $488 \mathrm{~nm}$. Cell cycle and apoptosis were determined and analyzed using ModFit software. The average of the percentage of each phase in the cell cycle is representative of three independent experiments.

Preparation of proteins. About $2 \times 10^{6}$ DBTRG cells in a $10-\mathrm{cm}$ dish were incubated with increased concentrations of curcumin, $0,8,16$, to $32 \mu \mathrm{g} / \mathrm{ml}$ for $24 \mathrm{~h}$ before cells were harvested by centrifugation. In addition, $\sim 2 \times 10^{6}$ cells in a $10-\mathrm{cm}$ dish were incubated with curcumin at $16 \mu \mathrm{g} / \mathrm{ml}$ concentration for increased durations of $0,12,24$, and $48 \mathrm{~h}$ before cells were harvested by centrifugation. Protein was extracted as described previously (13). Briefly, cell pellets were re-suspended in Pro-Prep buffer (Intron Biotechnology, Inc.) for $30 \mathrm{~min}$ at $4^{\circ} \mathrm{C}$. Lysates were immediately centrifuged at $13,000 \mathrm{x} \mathrm{g}$ for $20 \mathrm{~min}$ at $4^{\circ} \mathrm{C}$ and the supernatant was collected and stored at $-80^{\circ} \mathrm{C}$ until assay. Protein concentrations were measured with the Bradford method (14).

Western blotting for examining the effect of curcumin on the expression of cancer-related proteins. The samples were separated by sodium dodecylsulfate polyacrylamide (SDSPAGE) gel electrophoresis $(10,12.5$, and 15\%) (Bio-Rad Life Science Products, Hercules, CA, USA) as described (6). The SDS-separated proteins were followed by equilibration in transfer buffer [50 mM Tris, pH 9.0, $40 \mathrm{mM}$ glycine, $0.375 \%$ SDS, 20\% methanol and electro transferred to Immobilon-P Transfer Membrane (Millipore Corporation, Bedford, MA, USA)]. Then, the blot was blocked with a solution containing $5 \%$ nonfat dry milk in Tris-buffered saline $(10 \mathrm{mM}$ Tris, $150 \mathrm{mM} \mathrm{NaCl}$ Sigma Chemical Co.) containing 0.1\% Tween-20 for $30 \mathrm{~min}$. After that, the blot was washed and incubated with antibodies to caspase 3 (R\&D), Bax (Upstate), Bcl-2 (R\&D), p53 (Sigma), p21 (Upstate), m-TOR, Ras, PTEN, Akt, phosphated Akt, CDKN2A/p16 (Abcam), RB, and B-actin (Upstate) at $4{ }^{\circ} \mathrm{C}$ overnight. After incubating with anti-mouse peroxidase-conjugated antibody (Santa Cruz, CA, USA), the signal was visualized by enhanced chemiluminescence (ECL, Amersham Pharmacia Biotech). Detection of B-actin was used as an internal control in the data of Western blotting.

Giemsa stain. DBTRG cells were plated at a density of $1 \times 10^{4}$ cells/well and grown for $24 \mathrm{~h}$. Increased concentrations of curcumin, $0,8,16$, and $32 \mu \mathrm{g} / \mathrm{ml}$ were added and cells were cultured under two conditions, 24 or $48 \mathrm{~h}$. After removing the culture medium, dishes were washed twice with PBS and fixed with Carnoy's fixation solution (methanol and acetic 
A

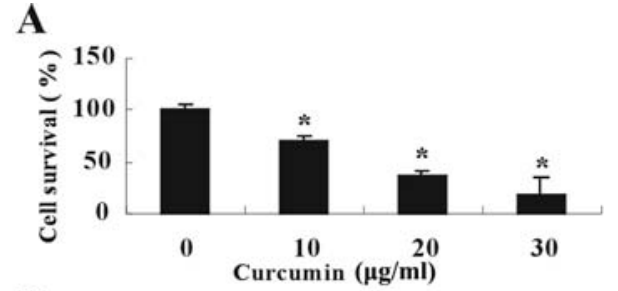

B

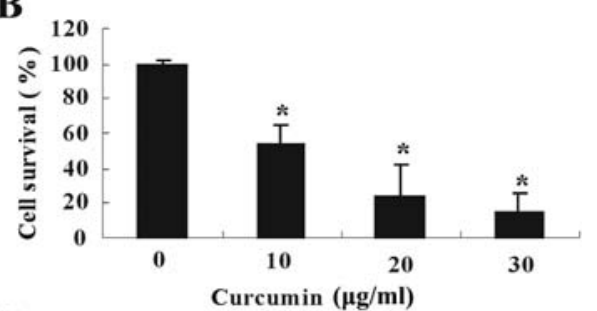

C

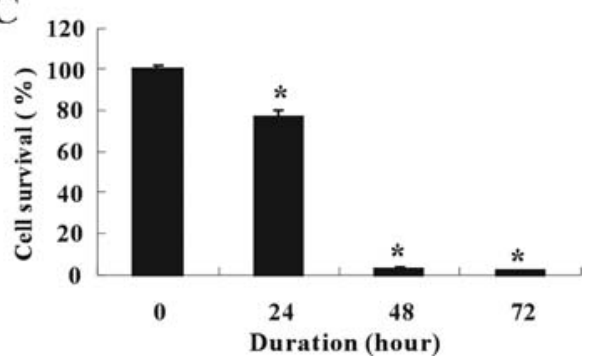

Figure 1. MTT assay of DBTRG cells treated by curcumin with increased doses and time intervals. A, DBTRG cells were treated with curcumin for $24 \mathrm{~h}$, and the therapeutic doses were $0,10,20$ and $30 \mu \mathrm{g} / \mathrm{ml}$. Cell-survival percentages were $100 \pm 4.9,69.1 \pm 4.7,37.9 \pm 4.5$, and $19.6 \pm 14.7 \%$, respectively. $\mathrm{B}, \mathrm{DBTRG}$ cells were treated with curcumin for $48 \mathrm{~h}$ with the same doses as in $\mathrm{A}$, and the cell-survival percentages were $100 \pm 2.7,53.6 \pm 11.7,24.6 \pm 17.2$, and $15.8 \pm 10.4 \%$ respectively. C, DBTRG cells were treated with $20 \mu \mathrm{g} / \mathrm{ml}$ curcumin for $0,24,48$, to $72 \mathrm{~h}$, and the cell-survival percentages were $100 \pm 2.4,79.9 \pm 3.7,3.2 \pm 0.4$ to $2.3 \pm 0.2 \%$, respectively. * Significant difference compared with control $(\mathrm{p}<0.05)$.

acid mixture, 3:1) for $10 \mathrm{~min}$ at room temperature. After, the fixation solution was removed. Dishes were washed twice with PBS again and added in 10\% Giemsa solution for staining. After 2-3 min incubation at room temperature, dishes were washed with PBS then examined under light microscope and photographed. In addition, the time-dependent model was performed with increased durations of 0,24 , and $48 \mathrm{~h}$.

Statistical analysis. Data were depicted with mean value \pm standard deviation. The untreated group $(0 \mu \mathrm{g} / \mathrm{ml})$ was set as the control group. The value (detected-protein/ $/$-actin) of the control group in Western blotting was adjusted to $100 \%$ and others (various concentrations and durations) were adjusted to the same ratio. The difference between the respective groups was compared with one-way ANOVA and Bonferroni post test, and p-values $<0.05$ were considered a significant difference for all tests.

\section{Results}

Cytotoxicity of curcumin on DBTRG cells by MTT assay. The MTT assay was performed in dose- and time-dependent modes. When the curcumin dose increased, the survival of DBTRG cells decreased gradually either after 24 or $48 \mathrm{~h}$ treatment by curcumin (Fig. 1A and B). The $\mathrm{IC}_{50}$ was $\sim 16.1 \mu \mathrm{g} / \mathrm{ml}$ after $24 \mathrm{~h}$ treatment and $11.2 \mu \mathrm{g} / \mathrm{ml}$ after $48 \mathrm{~h}$ treatment. In the timedependent mode, dramatic cell death developed after $48 \mathrm{~h}$ treatment of $20 \mu \mathrm{g} / \mathrm{ml}$ curcumin (Fig. 1C). These results indicate that curcumin exerts cytotoxic activity on DBTRG cells in a manner that is both time- and dose-dependent.

Morphological change of DBTRG cells treated with increased concentrations of curcumin. The morphological change of DBTRG cells was inspected after treatment with curcumin with increased concentrations and durations. Cells shrank from a flat and elongated shape to a small and unevenly round shape after treatment with curcumin. Adhesively viable cells decreased as the treatment dose increased (Fig. 2A-D) or treatment duration increased (Fig. 2E-H).

Cell cycle arrest induced by curcumin. The cell cycle of DBTRG cells was analyzed by flow cytometry after the treatment of curcumin with increased concentrations and durations. The cytometric histograms exhibited arrest of the cell cycle at the G2/M phase after treatment, compared with the

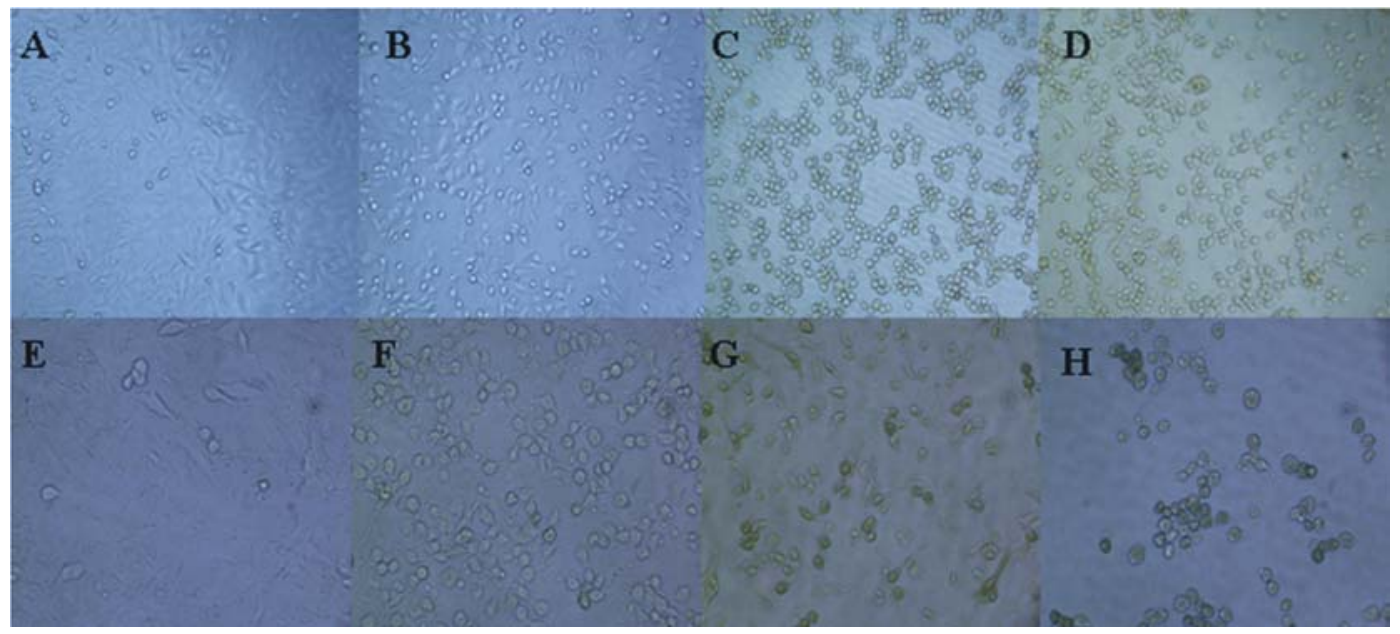

Figure 2. Photographs of DBTRG cells before and after treatment with curcumin. A-D, DBTRG cells treated with curcumin with increased concentration from A-D were $0,10,20$, to $30 \mu \mathrm{g} / \mathrm{ml}$, respectively (x100). E-H, DBTRG cells treated with $20 \mu \mathrm{g} / \mathrm{ml}$ curcumin with increased durations from E-H of 0,24 , 48 , and $72 \mathrm{~h}$, respectively $(\mathrm{x} 100)$. 

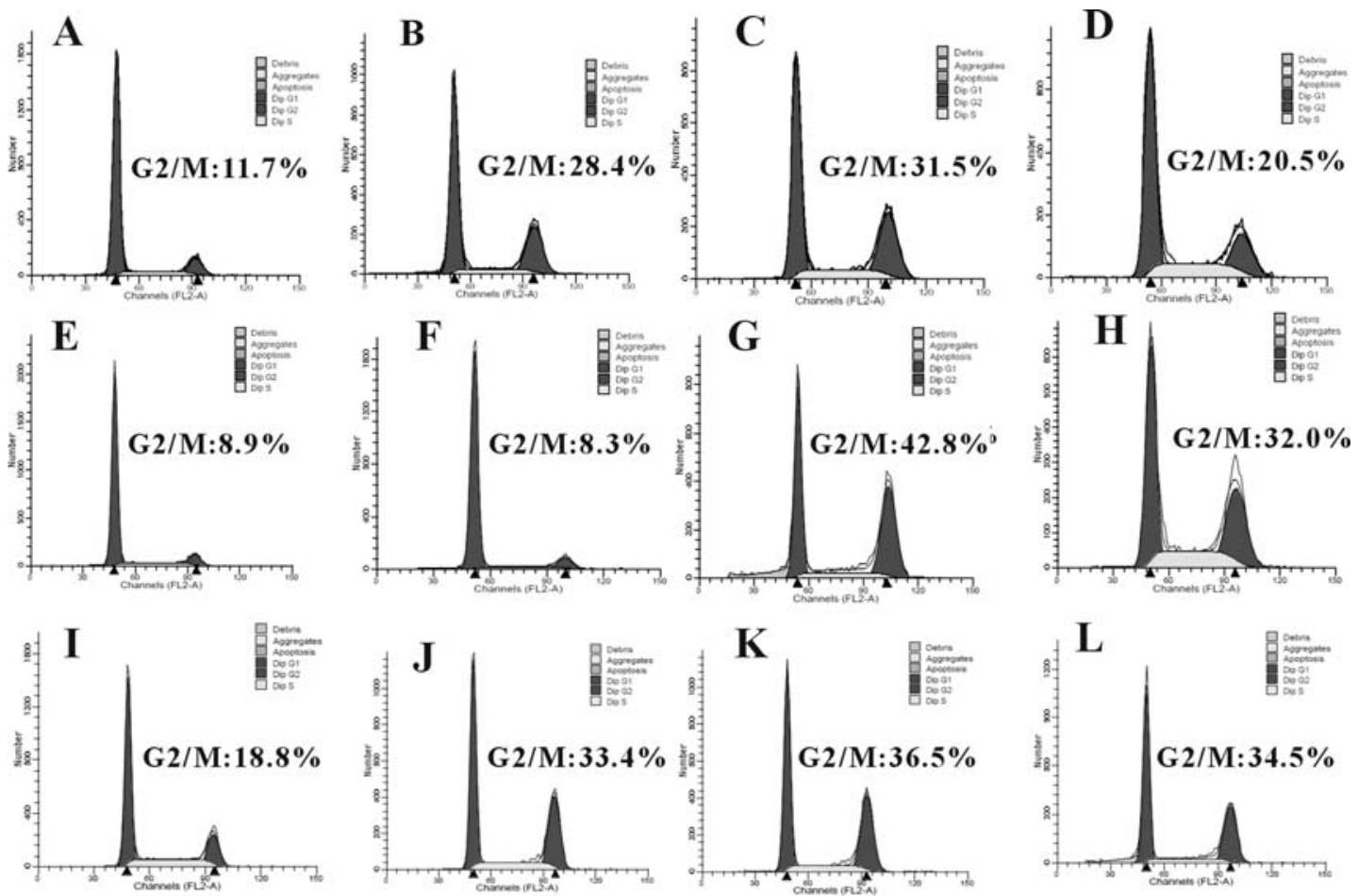

Figure 3. Flow cytometry of DBTRG cell cycle affected by the treatment of curcumin. A-D, DBTRG cells treated with various concentrations of curcumin, 0 (non-treated), 8, 16 and $32 \mu \mathrm{g} / \mathrm{ml}$ for $24 \mathrm{~h}$. The first pick waveform represents cells in G0/G1 phase and the secondary pick waveform represents cells in G2/M phase. E-H, DBTRG cells treated by various dosages of curcumin, 0 (non-treated), 8,16 , and $32 \mu \mathrm{g} / \mathrm{ml}$ for $48 \mathrm{~h}$. I-L, DBTRG treated with $16 \mu \mathrm{g} / \mathrm{ml}$ curcumin with increased durations, 0 (non-treated), 12, 24, and $48 \mathrm{~h}$.
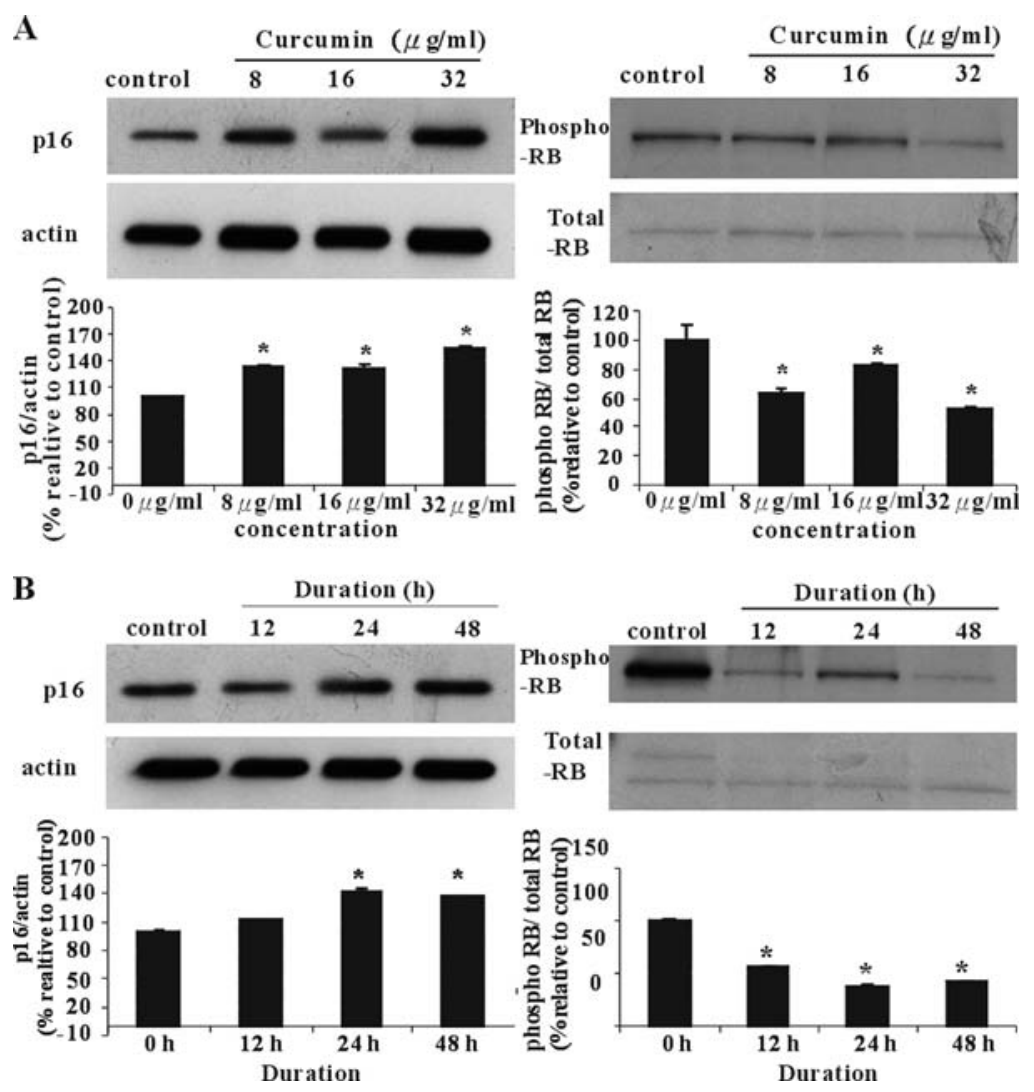

Figure 4. The effect of curcumin on the RB pathway. A, Left, Western blotting and histogram of CDKN2A/p16 with various dosages of curcumin; and right, phosphorylated RB. B, Western blotting and histogram of CDKN2A/p16 and phosphorylated RB treated with curcumin in various durations. ${ }^{*} \mathrm{p}<0.05$ compared with control. 
A
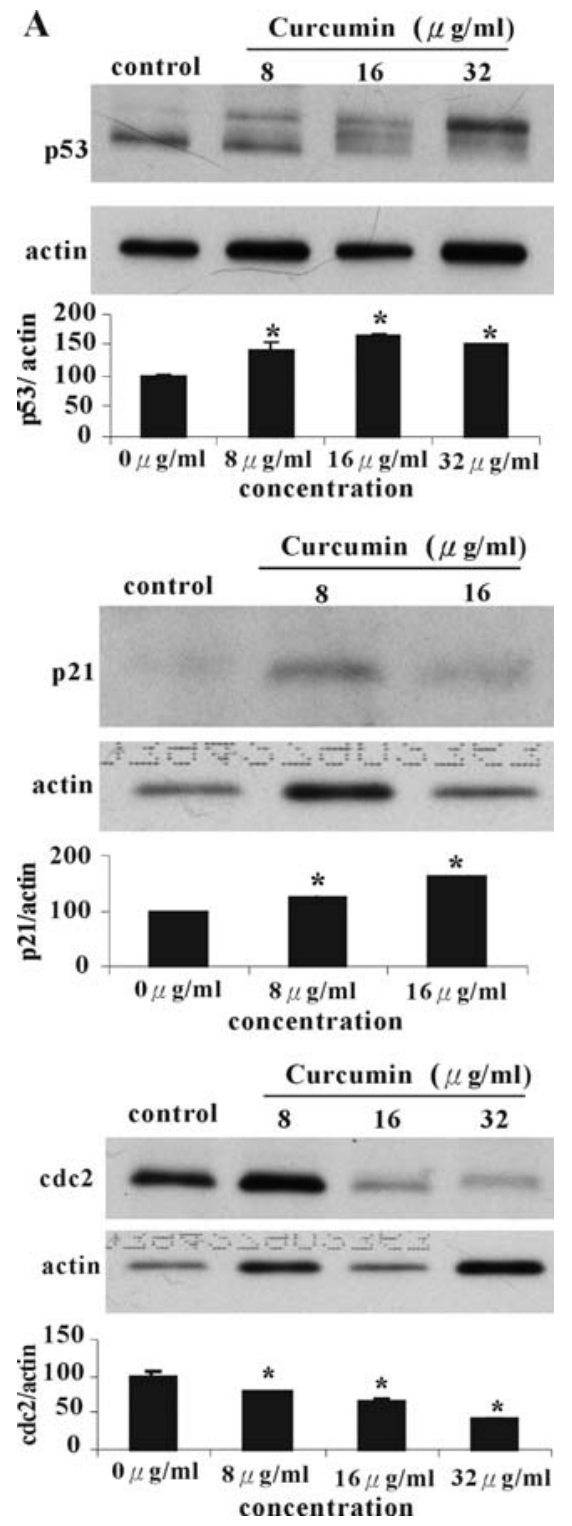
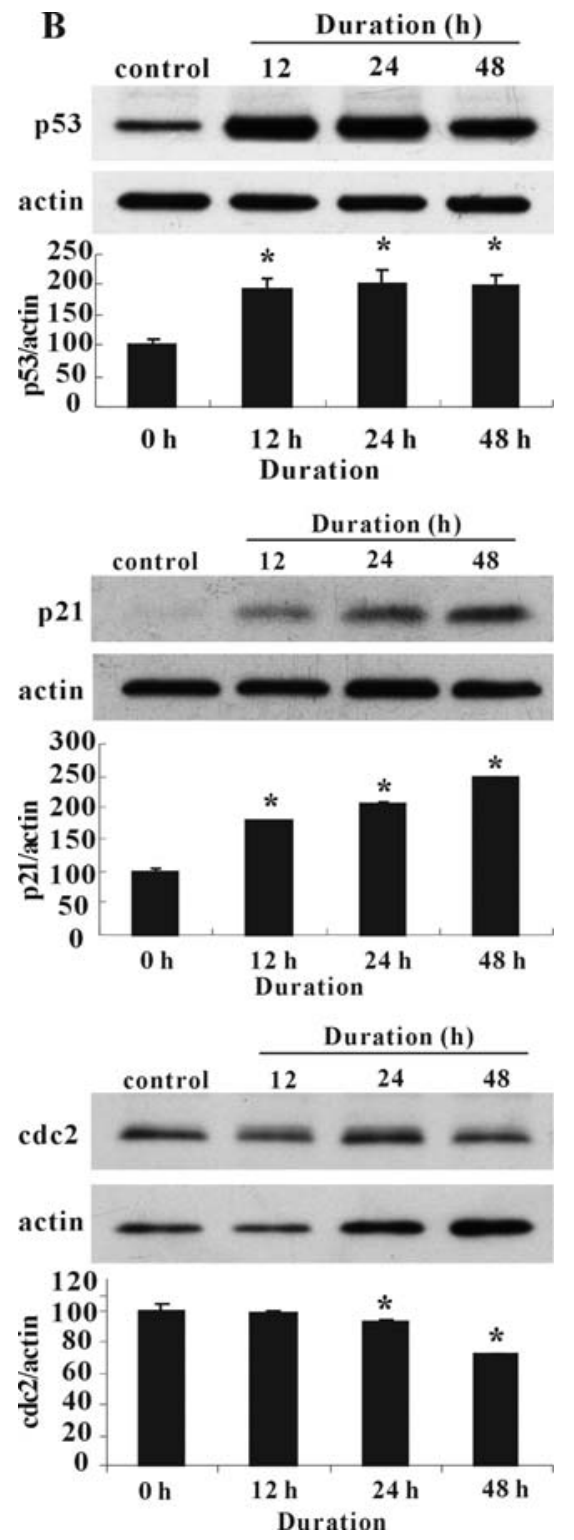

Figure 5. The effect of curcumin on the p53 pathway. A, Top, Western blotting and histogram of p53 in various dosages; middle, p21, and bottom, CDC2. B, Western blotting and histogram of p53, p21, and CDC2 treated in various durations. B, Western blotting and histogram of CDKN2A/p16 and phosphorylated $\mathrm{RB}$ treated with curcumin in various durations. * $\mathrm{p}<0.05$ compared with control.

non-treated, either in 24 or $48 \mathrm{~h}$ (Fig. 3A-H). The population of $\mathrm{G} 2 / \mathrm{M}$ phase cells increased 2-3-fold after treatment in the 24-h group and 3-4-fold in the 48-h group. When DBTRG cells were treated with $\mathrm{IC}_{50}(16 \mu \mathrm{g} / \mathrm{ml})$, the $\mathrm{G} 2 / \mathrm{M}$ phase cells increased nearly 2 -fold (I-L).

Effects of curcumin on aberrant-expression proteins in core signaling pathways of GBM. Three core signaling pathways were investigated, including the p53 pathway, RTK-RAS-PI3K pathway, and RB pathway. In total, 8 cancer-related proteins were evaluated for the effect of curcumin on their expression in DBTRG cells, which were p53 and p21 in the p53 pathway (Fig. 5), CDKN2A/p16 and RB in the RB pathway, and Ras, mTOR, PTEN, and ATK in the RTK-RAS-PI3K pathway (Fig. 6). In the RB pathway, CDKN2A/p16 exhibited upregulated and phosphorylated $\mathrm{RB}$ exhibited increased ratio to total RB after curcumin treatment either by increased treatment dosage or duration, which would result in arrest of the cancerous cell cycle at the G1/S stage and senescence or apoptosis of the cancerous cells subsequently (Fig. 3A and B). In the p53 pathway, p53 and p 21 were up-regulated by administration of curcumin either by increased treatment dosage or duration, which induced intrinsic apoptosis or arrest of the cancerous cell cycle at the G2/M stage (Fig. 4A and B). In the pathway of RTK-RAS-PI3K, PTEN showed no obvious influence by curcumin except in higher concentrations (32 $\mu \mathrm{g} / \mathrm{ml})$. Additionally, there was no significant difference of the ratio of phosphorylated Akt to total Akt. Ras and mTOR were not detected by Western blotting. From the above results, two of three core pathways of GBM, p53 and RB, are significantly inhibited by curcumin (Figs. 5 and 6).

Effects of curcumin on apoptosis-related proteins. Three apoptosis-related proteins were evaluated for the effect of curcumin on their expression from DBTRG cells, which included Bcl-2, Bax, and caspase 3. Curcumin slightly 

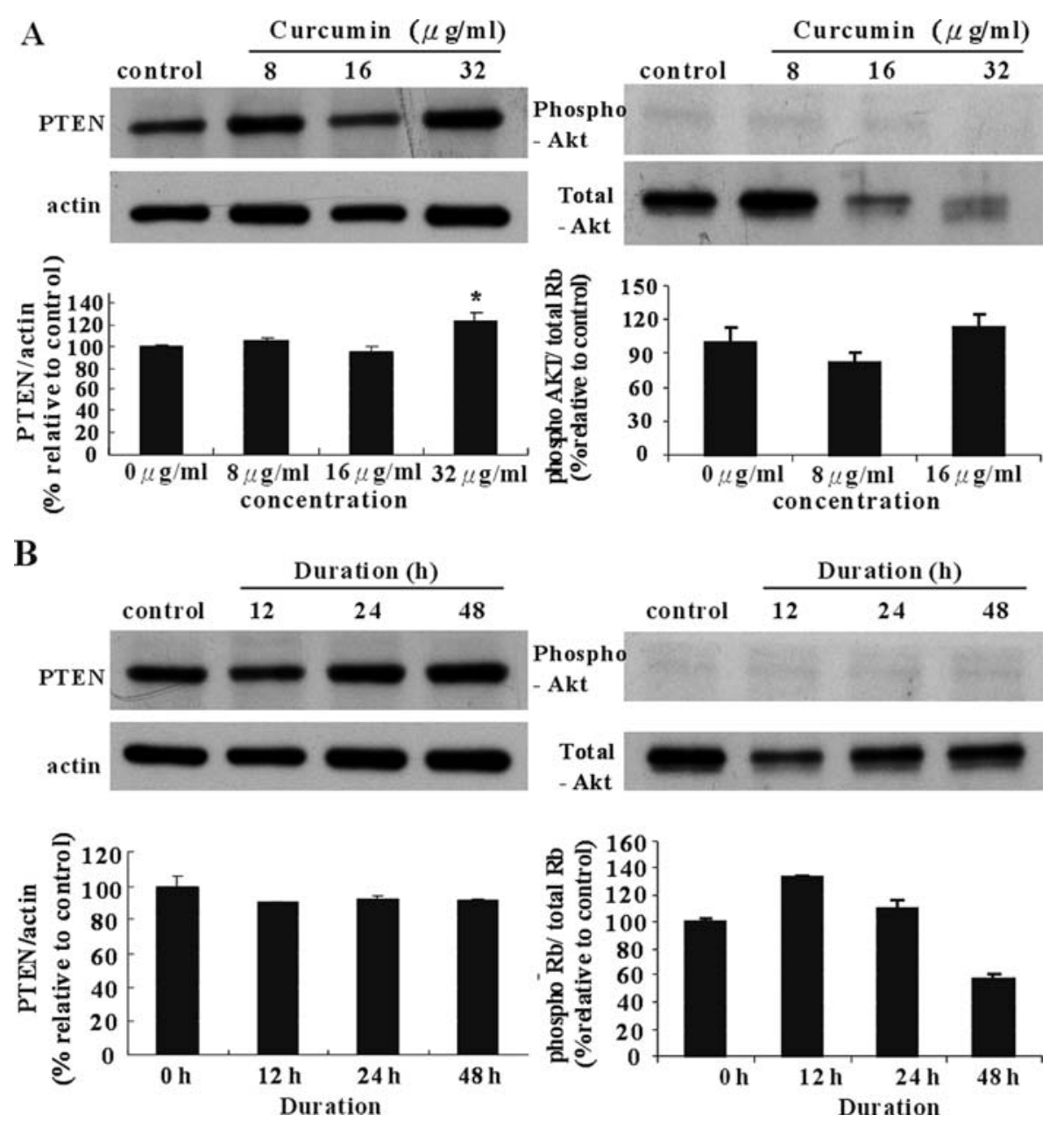

Figure 6. The effect of curcumin on RTK-Ras-PI3K pathway. A, Left, Western blotting and histogram of PTEN in various dosages; and right, phosphorylated Akt in various dosages. B, Western blotting and histogram of PTEN and phosphorylated Akt treated with curcumin in various durations. ${ }^{*}$ p $<0.05$ compared with control.

suppressed the expression of Bcl-2 but enhanced Bax expression apparently. To the downstream of the apoptosis pathway, caspase 3 was increased significantly, up to 2.5 -fold when the therapeutic duration was sustained for $>48 \mathrm{~h}$ (Fig. 7).

Apoptotic feature of DBTRG cells after curcumin treatment. Apoptosis of cells developed either after 24 or 48 h (Fig. 8A and B) treatment in increased-dosage mode, however, the morphological change became apparent when dosage went beyond the $\mathrm{IC}_{50}$ dose, $16 \mu \mathrm{g} / \mathrm{ml}$. With the increase of therapeutic time, the apoptotic feature was displayed more abundantly (Fig. 8C).

\section{Discussion}

The anti-cancer mechanisms of curcumin in a malignant brain tumor involve cell proliferation, growth, survival, and apoptosis. Nevertheless, most previously identified mechanisms are related to the apoptotic pathway (9). For example, Pratibha and colleagues examined and compared the efficacy of three curcuminoids through Bcl-2-mediated apoptosis. Surajit and colleagues demonstrated the activity of curcumin by inducing receptor- and mitochondria-mediated apoptotic pathways $(15,16)$. Besides the apoptotic pathway, Liu and colleagues claimed that curcumin exerts cancer resistance not through apoptosis but through arrest of the cell cycle at the G2/M phase and is mediated by up-regulation of p53 (8). Nevertheless, in this study, we explored the anti-cancer mechanism not only for apoptosis, but also documented the most prevalent genetic aberrance of GBM. It is apparent that curcumin exhibits a pleotropic anti-GBM phenomenon since curcumin could increase the $\mathrm{Bax} / \mathrm{Bcl}-2$ ratio to induce apoptosis and inhibit at least two cancer signaling pathways to induce arrest of the cell cycle at the same time.

In the Akt-related pathway, curcumin has been verified to inhibit phosphorylation of Akt in several cancer cell lines such as prostate cancer, leukemia, and even malignant brain tumor $(5,9,17)$. Nevertheless, the phosphorylation of Akt was not influenced by curcumin significantly in this experimental cell line. For further realization of this conflict, we checked the upstream of Akt in this cell line including EGFR, Ras, mTOR, and PTEN. It was concluded that the Ras and mTOR exhibited undetectable expression in Western blotting, the EGFR displayed no obvious change, and PTEN showed increased levels only in a highly therapeutic dosage of curcumin. This contradictory result is due to the polymorphic genetic aberrations in cancer cells and may play an important role in the resistance of cancer therapy. To survey the efficacy of a treating reagent through a comprehensive cancer genetic abnormality is required.

Arrest of the cell cycle at the G2/M phase is one of the effects of curcumin found in this study, which is in agreement 
$\mathbf{A}$
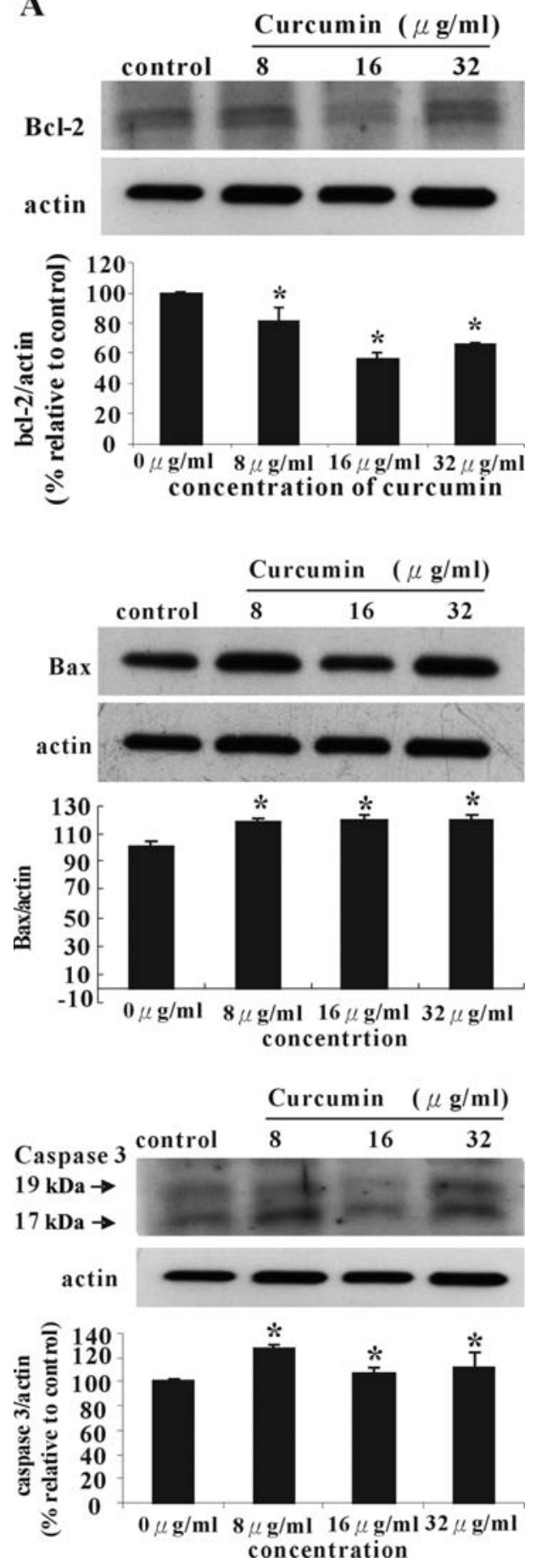

B
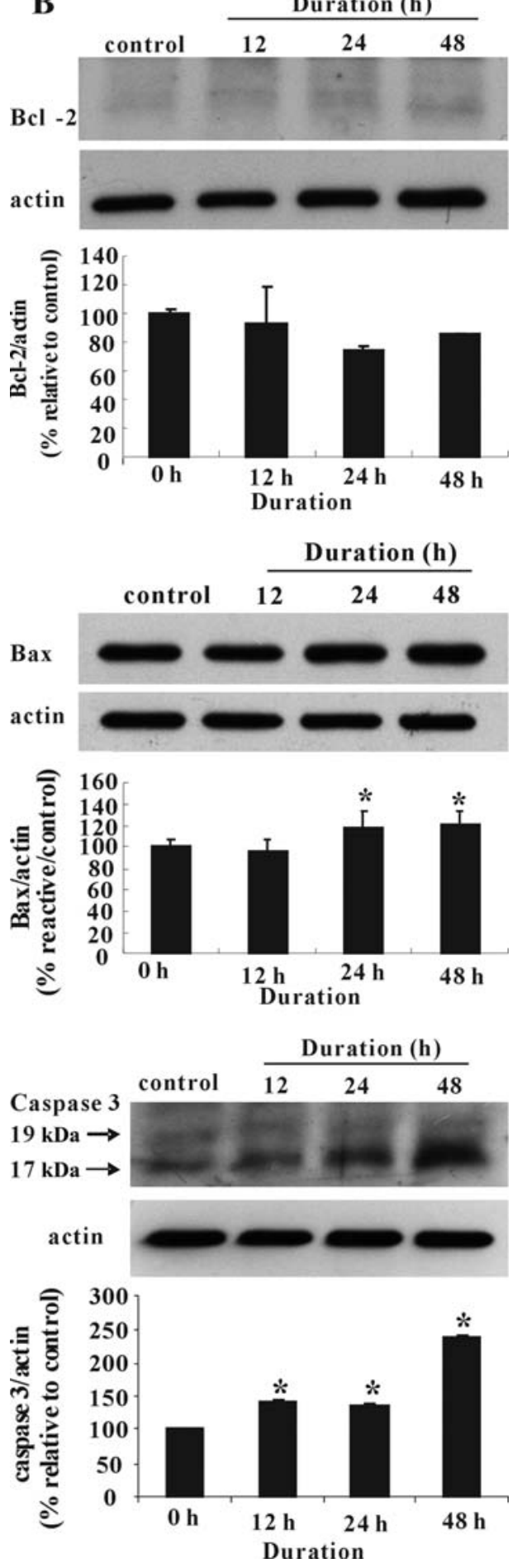

Figure 7. The influence of apoptosis-related proteins by curcumin. A, Top, Western blotting and histogram of Bcl-2 treated with curcumin with increased concentrations from $0,8,16$, and $32 \mu \mathrm{g} / \mathrm{ml}$; middle, Bax; and bottom, caspase 3. 17KD is the active form of caspase 3 which was measured for apoptotic efficacy. B, Left, Western blotting and histogram of Bcl-2 treated with curcumin with increased durations 0, 12, 24 , to 48 h; right, Bax; and lower, caspase 3. ${ }^{*} \mathrm{p}<0.05$ compared with control.

with our previous finding in breast cancer MDA-MB-231 cells, findings in human glioma U87 cells by Pratibha et al, and Liu et al in human glioma U251 cells $(4,7,16)$. This event was considered to be derived from up-regulation of $\mathrm{p} 53$, which has been identified as a common target of curcumin in different cancers. For insurance of p53 functioning in the check-point of G2/M, we further approve up-regulation of p21 and down-regulation of cdc 2 following the increasing of $\mathrm{p} 53$.
Within the core signaling pathway of GBM, the RB pathway could be inhibited by curcumin via up-regulation of CDKN2A/p16 and down-regulation of phosphorylated RB in this study. RB is a check-point of G1/S phase and is recognized as a tumor suppressor gene. Phosphorylation of RB induces dissociation of the RB-repressor complex and permits transcription of G1 gene for proceeding G1 to S phase (18). Inhibition of RB pathway and arrest of the cell cycle at $\mathrm{G} 1 / \mathrm{S}$ 


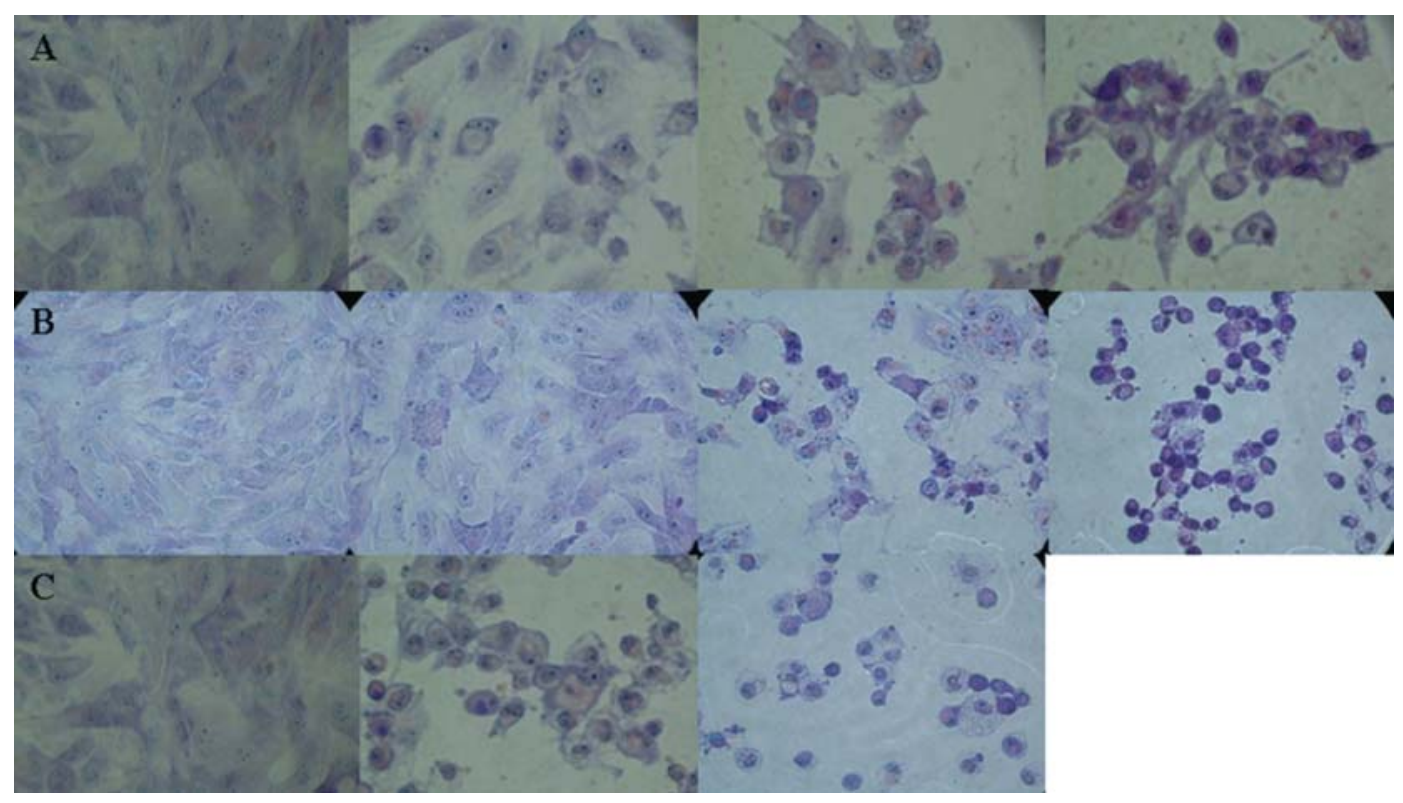

Figure 8. Giemsa stain of cancerous cells after curcumin treatment. A, DBTRG cells treated by curcumin for $24 \mathrm{~h}$ with $0,8,16$, and $32 \mu \mathrm{g} / \mathrm{ml}$ (left to right) (x400). B, DBTRG cells treated with curcumin for $48 \mathrm{~h}$ with the same dosages in A. C, DBTRG cells treated with $16 \mu \mathrm{g} / \mathrm{ml}$ curcumin for 0,24 , and $48 \mathrm{~h}$ (left to right) ( $\mathrm{x} 400$ ).

by curcumin has not been reported up to now except in one report, in which Shishir and colleagues demonstrated that

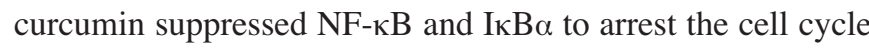
at the $\mathrm{G} 1 / \mathrm{S}$ phase by down-regulation of NF- $\mathrm{NB}$-regulated genes, and induced apoptosis by activation of caspases 9,7 , and 3 (19). In summary, curcumin promotes both checkpoints of the cell cycle, at least in this cell line model.

In this anti-GBM study, the efficacy of curcumin was investigated, including cytotoxicity of GBM cells, change of cell cycle of GBM, genetic expression of GBM signaling pathway, and the apoptotic pathway. The MTT assay revealed a dose- and time-dependent cytotoxic phenomenon of curcumin on GBM. Definite inhibition on the p53 and RB pathways by curcumin were validated via enhancing the expression of tumor suppressor genes and suppression of oncogenes. Curcumin shows potential benefits for treatment of GBM.

\section{References}

1. Ammon $\mathrm{H}$ and Wahl M: Pharmacology of Curcuma longa Planta Med 57: 1-7, 1991.

2. Aggarwal B, Kumar A and Bharti A: Anticancer potential of curcumin: preclinical and clinical studies. Anticancer Res 23 363-398, 2003.

3. Kunnumakkara A, Anand P and Aggarwal B: Curcumin inhibits proliferation, invasion, angiogenesis and metastasis of different cancers through interaction with multiple cell signaling proteins. Cancer Lett 269: 199-225, 2008.

4. Chen A, Xu J and Johnson A: Curcumin inhibits human colon cancer cell growth by suppressing gene expression of epidermal growth factor receptor through reducing the activity of the transcription factor Egr-1. Oncogene 25: 278-287, 2006.

5. Deeb D, Jiang H, Gao X, et al: Curcumin [1,7-Bis(4-hydroxy-3methoxyphenyl)-1-6-heptadine-3,5-dione; C21H20O6] sensitizes human prostate cancer cells to tumor necrosis factorrelated apoptosis-inducing ligand/Apo2L-induced apoptosis by suppressing nuclear factor- $\{$ kappa $\}$ B via inhibition of the prosurvival Akt signaling pathway. J Pharmacol Exp Ther 321: 616-625, 2007.

6. Chiu T and Su C: Curcumin inhibits proliferation and migration by increasing the Bax to $\mathrm{Bcl}-2$ ratio and decreasing NF- $\mathrm{Bp} 65$ expression in breast cancer MDA-MB-231 cells. Int J Mol Med 23: 469-475, 2009.
7. Dhandapani K, Mahesh V and Brann D: Curcumin suppresses growth and chemoresistance of human glioblastoma cells via AP-1 and NFkappaB transcription factors. J Neurochem 102: 522-538, 2007.

8. Liu E, Wu J, Cao W, et al: Curcumin induces G2/M cell cycle arrest in a p53-dependent manner and upregulates ING4 expression in human glioma. J Neurooncol 85: 263-270, 2007.

9. Purkayastha S, Berliner A, Fernando SS, et al: Curcumin blocks brain tumor formation. Brain Res 1266: 130-138, 2009.

10. Comprehensive genomic characterization defines human glioblastoma genes and core pathways. Nature 455: 1061-1068, 2008.

11. Wen PY and Kesari S: Malignant gliomas in adults. N Engl J Med 359: 492-507, 2008.

12. Li TM, Chen GW, Su CC, et al: Ellagic acid induced p53/p21 expression, G1 arrest and apoptosis in human bladder cancer T24 cells. Anticancer Res 25: 971-979, 2005.

13. Chen H, Hsieh W, Chang W and Chung J: Aloe-emodin induced in vitro $\mathrm{G} 2 / \mathrm{M}$ arrest of cell cycle in human promyelocytic leukemia HL-60 cells. Food Chem Toxicol 42: 1251-1257, 2004.

14. Bradford M: A rapid and sensitive method for the quantitation of microgram quantities of protein utilizing the principle of protein-dye binding. Anal Biochem 72: 248-254, 1976.

15. Karmakar S, Banik N and Ray S: Curcumin suppressed antiapoptotic signals and activated cysteine proteases for apoptosis in human malignant glioblastoma U87MG cells. Neurochem Res 32: 2103-2113, 2007

16. Luthra P, Kumar R and Prakash A: Demethoxycurcumin induces Bcl-2 mediated G2/M arrest and apoptosis in human glioma U87 cells. Biochem Biophys Res Commun 384: 420-425, 2009.

17. Ghosh AK, Kay NE, Secreto CR and Shanafelt TD: Curcumin inhibits prosurvival pathways in chronic lymphocytic leukemia B cells and may overcome their stromal protection in combination with EGCG. Clin Cancer Res 15: 1250-1258, 2009.

18. Martinez-Balbas M, Bauer U, Nielsen S, Brehm A and Kouzarides T: Regulation of E2F1 activity by acetylation. EMBO J 19: 662-671, 2000.

19. Shishodia S, Amin H, Lai R and Aggarwal B: Curcumin (diferuloylmethane) inhibits constitutive NF-kappaB activation, induces $\mathrm{G} 1 / \mathrm{S}$ arrest, suppresses proliferation, and induces apoptosis in mantle cell lymphoma. Biochem Pharmacol 70: 700-713, 2005. 